\title{
L to D and Back
}

\author{
Cyril W Smith* \\ Honorary Senior Lecturer, University of Salford, England
}

Submission: January 30, 2017; Published: July 07, 2017

*Corresponding author: Cyril W Smith, Honorary Senior Lecturer, University of Salford, England Salford M5 4WT, Email: cyril.smith@which.net

Abstract

A L- to D- isomerisation effect is demonstrated in the frequency signatures of two amino acids and in a yeast culture growth on exposure to $2.45 \mathrm{GHz}$ with the reverse $\mathrm{D}$ - to $\mathrm{L}$ - isomerisation on exposure to $62.5 \mathrm{MHz}$.

\section{Background}

Coherent frequencies [1-3] are involved in biological activity and can have a biological effect at the quantum level. I presented evidence in 1997 that the frequencies in a living system are those of a macroscopic quantum system4. Coherent frequencies are present as chemical frequency signatures arising from the interaction between a chemical and trace water [2], in acupuncture meridians and their target organs [3] and in homeopathic potencies [5].

Coherent frequencies can be imprinted into water, stored and erased [3]. Bio-information is carried on the magnetic vector potential component (A-field) of the magnetic field, its vector points in the direction of the current giving rise to it. The magnetic B-field component is equivalent to a momentum impulse and has a frequency imprinting function. A frequency is "remembered" in water through spin precession of protons and electrons imprinted from an oscillating A-field. The spin itself is determined by the geomagnetic field. If imprinted water is placed in a steel box so as to reduce the ambient magnetic field below a certain critical value determined by the size of the coherence domain, the imprint is erased by thermal agitation.

Chemicals acquire characteristic frequency signatures through hydrogen bonding to trace (vicinal) water. These disappear with sufficient dehydration. They arise through transitions between the chemical and water spectra in the far-infra-red spectral region and appear throughout the electromagnetic spectrum due to coherence.

The imprinting of frequencies into water involves proton and/or electron spin precession. Protons are otherwise called hydrogen ions and their concentration measured as $\mathrm{pH}$.
They are free to diffuse in water only. For example, I have not been able to imprint frequencies into concentrated hydrogen peroxide (H202). Frequencies can be imprinted into metals through electron spin resonance (ESR). For frequency imprints in water, the actual frequencies measured depends on the field configurations used to selectively excite the various resonances present.

It is a property of a coherent system such as water that that the constant parameter is the coherence length rather than the velocity of light. This gives rise to multiple frequencies each proportional to any velocity that the system can support, in particular the speed of light $(300 \mathrm{Mm} / \mathrm{s})$ and the speed of coherence diffusion $(\sim \mathrm{m} / \mathrm{s})$. This property of a coherent system interconnects effects throughout the electromagnetic spectrum - optical to microwave, to radio to low-frequencies.

\section{L- to D- Isomerisation}

Enzymes are built up from amino acids and are very sensitive to their tertiary structure. Lubec et al. [6] reported that amino acid isomerisation is changed from L- to D- following exposure in a microwave cooker $(2.45 \mathrm{GHz})$. They remarked that baby's bottle should not be warmed in the microwave if that is its only source of protein. Nowadays, this frequency is widespread in the environment being used for WiFi and many other purposes besides cooking. However, such frequency phenomena are usually biphasic and there is another frequency which will effect the D- to L- transformation.

Table 1, gives the original frequency imprints copied from the two amino acid specimens available in the L-chirality. After succussion next to a WiFi router or a coil excited at $2.45 \mathrm{GHz}$, 
different frequencies were measured and these were in the Dchirality. After succussion next to a coil excited at $62.5 \pm 0.2 \mathrm{MHz}$ they had returned to the L-chirality. A solution of L-fructose blocks the D-chirality and a solution of D-sucrose blocks the L-chirality.

Table 1: Frequency signatures of two Amino Acids Original, then after succussion in $2.45 \mathrm{GHz}$ and again after succussion in $62.5 \mathrm{MHz}$.

\begin{tabular}{|c|c|}
\hline L-Glutamine $>\mathbf{9 9 \%}$ & L-Asparagine $>\mathbf{9 9 \%}$ \\
\hline Frequency signature $\mathbf{~ H z}$ & Frequency signature $\mathbf{~ H z}$ \\
\hline $7.110 \times 10^{-2}$ & $6.003 \times 10^{-2}$ \\
\hline $7.502 \times 10^{-1}$ & $6.201 \times 10^{-1}$ \\
\hline $5.300 \times 10^{+2}$ & $5.002 \times 10^{+2}$ \\
\hline Succussed next to WiFi Router & Succussed next to $2.45 \mathrm{GHz}$ \\
\hline $4.202 \times 10^{-1}$ & $3.710 \times 10^{-1}$ \\
\hline $8.003 \times 10^{0}$ & $8.702 \times 10^{0}$ \\
\hline $6.416 \times 10^{+3}$ & $6.041 \times 10^{+3}$ \\
\hline Succussed next to $\mathbf{6 2 . 5} \mathbf{~ M H z}$ & Succussed next to $\mathbf{6 2 . 5} \mathbf{~ M H z}$ \\
\hline $7.110 \times 10^{-2}$ & $6.003 \times 10^{-2}$ \\
\hline $7.502 \times 10^{-1}$ & $6.201 \times 10^{-1}$ \\
\hline $5.300 \times 10^{+2}$ & $5.002 \times 10^{+2}$ \\
\hline
\end{tabular}

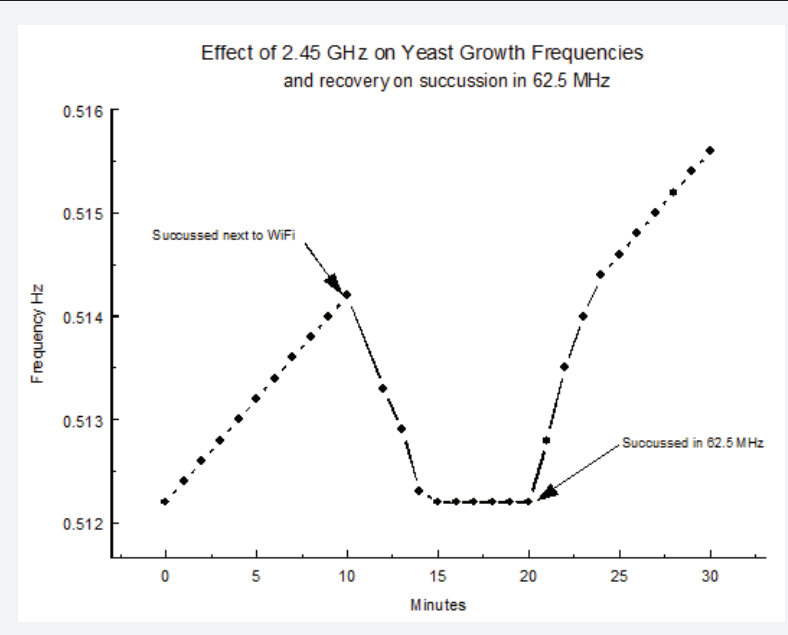

Figure 1: Effect of $2.45 \mathrm{GHz}$ on yeast growth frequencies and recovery on succussion in $62.5 \mathrm{MHz}$.
Figure 1 shows the change in the frequency signature of a yeast-sucrose culture following exposure to $2.45 \mathrm{GHz}$ and its recovery following exposure to $62.5 \mathrm{MHz}$.

These measurements were made in the field of a Caduceus coil which conveniently for this purpose gives only a single frequency to be measured. The Caduceus coil is wound like a solenoid for the first layer of wire but a second layer of wire is then wound back over it in the opposite sense. The resulting B-fields cancel so it is often called a 'non-inductive' coil. The tangential components of the A-fields also cancel but, radial A-field components remain.

\section{Conclusion}

These measurements demonstrate that a $0.3 \%$ coherent frequency can have a biological effect at the level of yeast growth and amino acid isomerisation. A person with chronic exposure to WiFi needs to be able to generate internally $62.5 \pm 0.2 \mathrm{MHz}$ on demand to avoid becoming deficient in L-amino acids.

\section{References}

1. Fröhlich H (1988) Theoretical Physics and Biology. In: Fröhlich H (Ed.), Biological Coherence and Response to External Stimuli. SpringerVerlag, Berlin, Germany, pp. 1-24.

2. Smith CW (2008) Fröhlich's Interpretation of Biology through Theoretical Physics. In: Hyland GJ, Rowlands P (Eds.), Herbert Fröhlich FRS: A physicist ahead of his time. $2^{\text {nd }}$ edn, University of Liverpool, England, pp. 107-154.

3. Smith CW (1988) Electromagnetic Effects in Humans. In: Fröhlich H (Ed.), Biological Coherence and Response to External Stimuli. SpringerVerlag, Berlin, Germany, pp. 205-232,

4. Smith CW (1998) Is a living system a macroscopic quantum system? Frontier Perspectives 7(1): 9-15,

5. Smith CW (2008) Homeopathy - How It Works and How It Is Done. Chapters 1-7, Chapter 8.

6. Lubec G, Wolf C, Bartosch B (1989) Aminoacid Isomerisation and Microwave Exposure. The Lancet 334(8676): 1392-1393. 